\title{
Joint MIMO Channel Tracking and Symbol Decoding
}

\author{
B. Balakumar and T. Kirubarajan \\ McMaster University \\ Canada
}

\section{Introduction}

The objective of fourth generation (4G) wireless communication systems is to achieve broadband connectivity anytime, anywhere and between anything. It is anticipated that in the next two decades, this is likely to be achieved by employing multiple antennas at the base station (service provider) as well as at the end user devices. In this regard, the past decade has seen an explosion of interest in multi antenna systems, especially, multi-input multi-output (MIMO) communication systems. MIMO communication systems provide a promising approach to deliver higher data throughput without the need for increased power and bandwidth. Space-time coding adds time as another dimension to the diversity, in addition to the spatial diversity achieved by multiple antennas in MIMO system.

Among different space-time coding schemes presented in the literature, orthogonal spacetime block codes (OSTBCs, Alamouti, 1998) are of particular interest since they achieve full diversity at a lower receiver complexity. Indeed, many practical systems and standards such as WiFi and WiMAX have already adopted OSTBC for MIMO communications. The primary interest of this Chapter is to delve into the receiver aspect of the MIMO system where continuous interest has been shown in developing blind-adaptive decoding algorithms. Blind decoding algorithms improve data throughput by enabling the system designer to replace training symbols with data. On the other hand, considering the fact that the wireless end user environment is becoming increasingly mobile, adaptive algorithms have the ability to improve the performance of a system regardless of whether it is a blind system or training based one. The difficulty faced by blind and adaptive algorithms is that they are generally computationally intense. Further, the possible use of higher frequency carriers and the appeal of broadband communication for a wide variety of applications as well as the emergence of newer technologies such as cooperative communications have been contributing to the growing interest in a general $M \times N$ MIMO system over the $2 \times 1$ Alamouti's scheme which was the subject of intense research in the past decade. Hence, any blind-adaptive algorithm should be computationally efficient before they can be implemented in practical systems. This Chapter presents blind decoding algorithms that are adaptive in nature as well as computationally efficient for any $M \times N$ MIMO system.

In (Liu et al., 2002), Kalman filtering has been studied in application to channel tracking for MIMO communication systems. The method proposed in (Liu et al., 2002) is based on two assumptions. First, the underlying space-time coding scheme is based on Alamouti code Source: Kalman Filter: Recent Advances and Applications, Book edited by: Victor M. Moreno and Alberto Pigazo, ISBN 978-953-307-000-1, pp. 584, April 2009, I-Tech, Vienna, Austria 
(Alamouti, 1998), and therefore its application is limited to the case of two transmit antennas. Second, the channel is assumed to be time-varying during the transmission of each block. The latter assumption implies that the linear ML receiver is optimal in a mean sense (Liu et al., 2002). Kalman filtering has been applied to the problem of MIMO channel tracking in several other research reports (Schafhuber et al., 2003). Also, in (Coon et al., 2005), a frequency domain equalization method has been proposed for single carrier MIMO systems. Particle filtering has also been used in other studies (Haykin et al., 2004) for MIMO channel tracking. In (AlNauffouri et al., 2004), a Kalman filtering approach has been used in the maximization step of an expectation-maximization (EM) method to track the frequency selective MIMO channel when the underlying code is an OSTBC and when an orthogonal frequency division multiplexing (OFDM) is used.

This chapter also focuses on the MIMO channel tracking and data decoding algorithms (Balakumar et al., 2007) that are a) suitable for any $M \times N$ MIMO system and b) computationally efficient to be able to implement in practical systems. By considering a class of MIMO systems where OSTBCs are used as the underlying space-time coding schemes and assuming a fixed channel during the transmission of each block of data a two-step channel tracking algorithm is developed. In the first step, Kalman filtering is used at the beginning of each block to obtain an initial channel estimate for that block based on the channel estimate obtained for previous block. In the second step, to improve the quality of the channel estimate obtained by Kalman filtering, a simple iterative channel estimation technique is proposed. This iterative method is in fact a decision-directed algorithm and it consists of sequential use of a linear receiver and a linear channel estimator. In addition, it is shown that, due to specific properties of orthogonal space-time block codes, both the Kalman filter and the decision-directed algorithm can be significantly simplified.

\section{Background}

Consider a MIMO system with $N$ transmit and $M$ receive antennas. We consider a block transmission scheme and assume that within the block period $T$ the channel is fixed, i.e., the channel is assumed to be quasi-static. However, between different blocks the channel can change. As such, the $n$th received block can be written as

$$
\mathbf{Y}(n)=\mathbf{X}(n) \mathbf{H}(n)+\mathbf{V}(n)
$$

where $\mathbf{Y}(n)$ is the $T \times M$ matrix of the received signals, $\mathbf{X}(n)$ is the $T \times N$ matrix of transmitted signals, $\mathbf{V}(n)$ is the $T \times M$ matrix of noise, and $\mathbf{H}(n)$ is the $N \times M$ channel matrix during the $n$th block period. The noise $\mathbf{V}(n)$ is assumed to be zero-mean complex Gaussian and both spatially and temporally white with variance $\sigma_{v}^{2} / 2$ per real dimension.

In space-time block coding, the matrix $\mathbf{X}(n)$ is a mapping that transforms a block of complex symbols to a $T \times N$ complex matrix. Hence, we hereafter replace $\mathbf{X}(n)$ with $\mathbf{X}(\mathbf{s}(n))$ where $s(n)$ is the $n$th symbol vector of length $K$. Let us define $\mathbf{s}(n)$ as

$$
\mathbf{s}(n)=\left[s_{1}(n) s_{2}(n) \cdots s_{K}(n)\right]^{T}
$$

where $(\cdot)^{T}$ denotes the transpose operator. The $T \times N$ matrix $\mathbf{X}(s(n))$ is called an OSTBC (Alamouti, 1998; Tarokh et al., 1999) if i) all elements of $\mathbf{X}(\mathbf{s}(n))$ are linear functions of the 
$K$ complex variables $s_{1}(n), s_{2}(n), \ldots, s_{K}(n)$ and their complex conjugates, ii) and if, for any arbitrary $\mathbf{s}(n), \mathbf{X}(\mathbf{s}(n))$ satisfies

$$
\mathbf{X}^{H}(\mathbf{s}(n)) \mathbf{X}(\mathbf{s}(n))=\|\mathbf{s}(n)\|^{2} \mathbf{I}_{N}
$$

where $\mathbf{I}_{N}$ is the $N \times N$ identity matrix, $\|\cdot\|$ is the Euclidean norm, and $(\cdot)^{H}$ denotes Hermitian transpose.

It follows from the definition of OSTBC that matrix $\mathbf{X}(\mathbf{s}(n))$ can be written as

$$
\mathbf{X}(\mathbf{s}(n))=\sum_{k=1}^{K}\left(\mathbf{C}_{k} \operatorname{Re}\left\{s_{k}(n)\right\}+\mathbf{D}_{k} \operatorname{Im}\left\{s_{k}(n)\right\}\right)
$$

where $\operatorname{Re}\{\}$ and $\operatorname{Im}\{\cdot\}$ denote the real and imaginary parts, respectively, and $C_{k}$ and $D_{k}$ matrices are defined as ${ }^{1}$

$$
\begin{aligned}
\mathbf{C}_{k} & =X\left(\mathbf{u}_{k}\right) \\
\mathbf{D}_{k} & =X\left(j \mathbf{u}_{k}\right)
\end{aligned}
$$

where $\mathbf{u}_{k}$ is the $k$ th column of identity matrix $\mathbf{I}_{K}$ and $j=\sqrt{-1}$. Let us define the "underline" operator for a matrix $\mathbf{P}$ as

$$
\underline{\mathbf{P}} \triangleq\left[\begin{array}{l}
\operatorname{vec}\{\operatorname{Re}(\mathbf{P})\} \\
\operatorname{vec}\{\operatorname{Im}(\mathbf{P})\}
\end{array}\right]
$$

where vec \{\} refers to the vectorization operator stacking all the columns of a matrix on top of each other. Using (4) and (7), one can re-write (1) as (Gharavi-Alkhansari \& Gershman, 2005)

$$
\mathbf{y}(n) \triangleq \underline{\mathbf{Y}(n)}=\mathbf{A}(\mathbf{H}(n)) \mathbf{s}_{n}+\mathbf{v}_{n}
$$

where $\mathbf{s}_{n} \triangleq \underline{\mathbf{s}(n)}, \mathbf{v}_{n} \triangleq \underline{\mathbf{V}(n)}$ and the $2 M T \times 2 K$ real matrix $\mathbf{A}(\mathbf{H}(n))$ is given by

$$
\mathbf{A}(\mathbf{H}(n))=\left[\underline{\mathbf{C}_{1} \mathbf{H}(n)} \ldots \underline{\mathbf{C}_{K} \mathbf{H}(n)} \underline{\mathbf{D}_{1} \mathbf{H}(n)} \ldots \underline{\mathbf{D}_{K} \mathbf{H}(n)}\right] .
$$

It has been shown that for any channel matrix $\mathbf{H}(n)$, the matrix $A(\mathbf{H}(n))$ satisfies the socalled decoupling property, i.e., its columns are orthogonal to each other and have identical norms (Larsson \& Stoica, 2003). More specifically, it satisfies

$$
\mathbf{A}^{T}(\mathbf{H}(n)) \mathbf{A}(\mathbf{H}(n))=\|\mathbf{H}(n)\|_{F}^{2} \mathbf{I}_{2 K}
$$

where $\|\cdot\|_{F}$ denotes the Frobenius norm. Let us define the $2 M N \times 1$ time-varying channel vector $\mathbf{h}(n)$ as $\mathbf{h}(n) \triangleq \mathbf{H}(n)$. With a small abuse of notation, we hereafter replace $A(\mathbf{H}(n))$ with $A(\mathbf{h}(n))$. Therefore, we rewrite (10) as

IIn fact, any OSTBC is completely defined by the set of matrices $\left\{\mathbf{C}_{k}, \mathbf{D}_{k}\right\}_{k=1}^{K}$. 


$$
\mathbf{A}^{T}(\mathbf{h}(n)) \mathbf{A}(\mathbf{h}(n))=\|\mathbf{h}(n)\|^{2} \mathbf{I}_{2 K}
$$

Since $\mathbf{A}(\mathbf{h}(n))$ is linear in $\mathbf{h}(n)$, there exists a unique $4 K M T \times 2 M N$ matrix $\boldsymbol{\Phi}$ such that $v e c\{\mathbf{A}(\mathbf{h}(n))\}=\boldsymbol{\Phi} \mathbf{h}(n)$ where $\boldsymbol{\Phi}$ is a $4 K M T \times 2 M N$ matrix whose $k$ th column is given by

$$
[\boldsymbol{\Phi}]_{k}=\operatorname{vec}\left\{\mathbf{A}\left(\mathbf{e}_{k}\right)\right\} .
$$

Here, $[\cdot]_{k}$ denotes the $k$ th column of a matrix and $\mathbf{e}_{k}$ is the $k$ th column of identity matrix $\mathbf{I}_{2 M N}$. Note that matrix $\boldsymbol{\Phi}$ can be written as $\boldsymbol{\Phi}=\left[\boldsymbol{\Phi}_{1}^{T} \boldsymbol{\Phi}_{2}^{T} \ldots \boldsymbol{\Phi}_{2 K}^{T}\right]^{T}$ where each sub-matrix $\boldsymbol{\Phi}_{k}$, $(k=1, \ldots 2 K)$ describes the linear relationship between the $k$ th column of $\mathbf{A}(\mathbf{h}(n))$ and $\mathbf{h}(n)$, $[\mathbf{A}(\mathbf{h})]_{k}=\boldsymbol{\Phi}_{k} \mathbf{h}(n)$.

Given the channel vector $\mathbf{h}(n)$, the optimal ML decoder for OSTBCs consists of a linear receiver followed by a symbol-by-symbol decoder (Ganesan \& Stoica, 2001). Indeed, the linear receiver computes $\hat{\mathbf{s}}_{n}$, the estimate of $\mathbf{s}_{n}$ as

$$
\hat{\mathbf{s}}_{n}=\frac{1}{\|\mathbf{h}(n)\|^{2}} \mathbf{A}^{T}(\mathbf{h}(n)) \mathbf{y}_{n} .
$$

Then, the symbol-by-symbol decoder builds the estimate $\mathbf{s}(n)$, of vector $\mathbf{s}(n)$ as

$$
\hat{\mathbf{s}}(n)=\left[\mathbf{I}_{K} j \mathbf{I}_{K}\right] \hat{\mathbf{s}}_{n}
$$

The $k$ th element of $\mathbf{s}(n)$ is compared with all points in the constellation corresponding to $\mathbf{s}_{k}(n)$ and the closest point in this constellation to the $k$ th element of $\mathbf{s}(n)$ is accepted as the $k$ th decoded symbol.

Note however that implementation of the ML decoder requires the knowledge of the timevarying channel. If the channel is fixed, one can use training to estimate the channel in a non-blind fashion. However, in practice, the channel is time-varying, and hence tracking of the MIMO channel is required. Recently, blind channel estimation has been studied in the literature (see for example (Shahbazpanahi et al., 2005)). The blind channel estimation of (Shahbazpanahi et al., 2005) is based on the assumption that the channel is fixed, and hence, it is not applicable to time-varying channels.

Without assuming any model for the MIMO channel, the problem of joint channel tracking and symbol detection is ill-posed. Fortunately, in many practical scenarios, the wireless channels can be modeled with a few parameters. It has been shown in (Wang \& Chang, 1996) that the first-order AR model can be used as a sufficiently precise method to describe the time-varying behavior of wireless channels. Based on this model, we assume that the channel variation between adjacent blocks is modeled as a first order autoregressive (AR) model, i.e.,

$$
\mathbf{H}(n)=\alpha \mathbf{H}(n-1)+\mathbf{W}(n)
$$

where $\mathbf{W}(n)$ is an $N \times M$ noise matrix that is assumed to be zero-mean complex Gaussian with independent entries and variance of $\sigma_{w}^{2} / 2$ per real dimension. This implies that $\mathbf{W}(n)$, and consequently $\mathbf{H}(n)$, are zero-mean wide-sense stationary processes. The parameter $\alpha$ is a complex scalar that can be estimated using the method of (Tsatsanis et al., 1999), and 
hence, it is herein assumed to be known. The noise variance $\sigma_{w}^{2}$ and $\alpha$ are related as $\sigma_{w}^{2}=\sigma_{h}^{2}\left(1-|\alpha|^{2}\right)$ where $\sigma_{h}^{2}$ is the variance of each element of $\mathbf{H}(n)$ and $|\cdot|$ denotes the amplitude of a complex number.

\section{Kalman filter based channel tracking}

In this Section, the problem of channel tracking via Kalman filtering is addressed. Subsequently, a two-step channel tracking algorithm is proposed. In the first step of this algorithm, Kalman filtering is used to obtain an initial channel estimate for each block based on the channel estimates obtained for the previous blocks. In the second step, the soobtained initial channel estimate is refined using an iterative decision-directed technique, which involves a linear ML channel estimator based on the decoded symbols. In fact, the linearity of such an ML channel estimator follows from the interesting properties of OSTBCs. It is also demonstrated that due to the specific structure of OSTBCs, Kalman filtering based channel tracking can be significantly simplified.

To derive the two-step channel tracking algorithm, we rewrite (8) as

$$
\mathbf{y}_{n}=\mathbf{B}\left(\mathbf{s}_{n}\right) \mathbf{h}(n)+\mathbf{v}_{n}
$$

where the $2 M T \times 2 M N$ real matrix $\mathbf{B}\left(\mathbf{s}_{n}\right)$ is defined as

$$
\mathbf{B}\left(\mathbf{s}_{n}\right) \triangleq\left[A\left(\mathbf{e}_{1}\right) \mathbf{s}_{n} \quad A\left(\mathbf{e}_{2}\right) \mathbf{s}_{n} \ldots A\left(\mathbf{e}_{2 M N}\right) \mathbf{s}_{n}\right]
$$

and $\mathbf{e}_{k}$, as defined earlier, is the $k$ th column of the identity matrix $\mathbf{I}_{2 M N}$. The following Lemma plays an important role in simplifying the forthcoming Kalman filtering algorithm.

Lemma 1: The matrix $\mathbf{B}\left(s_{n}\right)$ satisfies

$$
\mathbf{B}^{T}\left(\mathbf{s}_{n}\right) \mathbf{B}\left(\mathbf{s}_{n}\right)=\|\mathbf{s}(n)\|^{2} \mathbf{I}_{2 M N}
$$

Proof: We first show that the sub-matrices $\left\{\boldsymbol{\Phi}_{k}\right\}_{k=1}^{2 K}$ satisfy the following equations:

$$
\boldsymbol{\Phi}_{l}^{T} \boldsymbol{\Phi}_{m}= \begin{cases}\mathbf{I}_{2 M N} & \text { if } l=m, \\ -\boldsymbol{\Phi}_{m}^{T} \boldsymbol{\Phi}_{l} & \text { if } l \neq m .\end{cases}
$$

To prove (19), we use the decoupling property in (11). Indeed, for any channel vector $\mathbf{h}$, the decoupling property in (11) implies that

$$
[A(\mathbf{h})]_{l}^{T}[A(\mathbf{h})]_{l}=\|\mathbf{h}\|^{2}
$$

or

$$
\mathbf{h}^{T} \boldsymbol{\Phi}_{l}^{T} \boldsymbol{\Phi}_{l} \mathbf{h}=\mathbf{h}^{T} \mathbf{h}
$$

Since (21) holds true for any $\mathbf{h}$ and because $\boldsymbol{\Phi}_{l}^{T} \boldsymbol{\Phi}_{l}$ is a symmetric matrix, we conclude that $\boldsymbol{\Phi}_{l}^{T} \boldsymbol{\Phi}_{l}=\mathbf{I}_{2 M N}$. To prove the second part of (19), based on the fact that different columns of $A(\mathbf{h})$ are orthogonal to each other, we can write 


$$
\left.\begin{array}{l}
{[A(\mathbf{h})]_{l}^{T}[A(\mathbf{h})]_{m}=\mathbf{h}^{T} \boldsymbol{\Phi}_{l}^{T} \boldsymbol{\Phi}_{m} \mathbf{h}=0} \\
{[A(\mathbf{h})]_{m}^{T}[A(\mathbf{h})]_{l}=\mathbf{h}^{T} \boldsymbol{\Phi}_{m}^{T} \boldsymbol{\Phi}_{l} \mathbf{h}=0}
\end{array}\right\} \Rightarrow \mathbf{h}^{T}\left(\boldsymbol{\Phi}_{l}^{T} \boldsymbol{\Phi}_{m}+\boldsymbol{\Phi}_{m}^{T} \boldsymbol{\Phi}_{l}\right) \mathbf{h}=0
$$

Since (22) holds true for any vector $\mathbf{h}$ and since $\boldsymbol{\Phi}_{l}^{T} \boldsymbol{\Phi}_{m}+\boldsymbol{\Phi}_{m}^{T} \boldsymbol{\Phi}_{l}$ is a symmetric matrix, we conclude that $\boldsymbol{\Phi}_{l}^{T} \boldsymbol{\Phi}_{m}+\boldsymbol{\Phi}_{m}^{T} \boldsymbol{\Phi}_{l}=0$. This completes the proof of (19).

We now use (19) to prove (18). To do so, we note that

$$
\mathbf{B}^{T}\left(\mathbf{s}_{n}\right) \mathbf{B}\left(\mathbf{s}_{n}\right)=\left[\begin{array}{ccc}
\mathbf{s}_{n}^{T} A^{T}\left(\mathbf{e}_{1}\right) A\left(\mathbf{e}_{1}\right) \mathbf{s}_{n} & \cdots & \mathbf{s}_{n}^{T} A^{T}\left(\mathbf{e}_{1}\right) A\left(\mathbf{e}_{2 M N}\right) \mathbf{s}_{n} \\
\mathbf{s}_{n}^{T} A^{T}\left(\mathbf{e}_{2}\right) A\left(\mathbf{e}_{1}\right) \mathbf{s}_{n} & \cdots & \mathbf{s}_{n}^{T} A^{T}\left(\mathbf{e}_{2}\right) A\left(\mathbf{e}_{2 M N}\right) \mathbf{s}_{n} \\
\vdots & \ddots & \vdots \\
\mathbf{s}_{n}^{T} A^{T}\left(\mathbf{e}_{2 M N}\right) A\left(\mathbf{e}_{1}\right) \mathbf{s}_{n} & \cdots & \mathbf{s}_{n} A^{T}\left(\mathbf{e}_{2 M N}\right) A\left(\mathbf{e}_{2 M N}\right) \mathbf{s}_{n}
\end{array}\right]
$$

where we have used (17). Note also that

$$
\mathbf{s}_{n}^{T} \underbrace{A^{T}\left(\mathbf{e}_{l}\right) A\left(\mathbf{e}_{l}\right)}_{\left\|\mathbf{e}_{l}\right\|^{\|^{2}} \mathbf{I}_{2 K}} \mathbf{s}_{n}=\left\|\mathbf{s}_{n}^{T}\right\|^{2}
$$

which follows from the decoupling property. For $l \neq m$, the following set of equalities holds true:

$$
\begin{aligned}
\mathbf{s}_{n}^{T} A^{T}\left(\mathbf{e}_{l}\right) A\left(\mathbf{e}_{m}\right) \mathbf{s}_{n} & =\sum_{r=1}^{2 K} \sum_{s=1}^{2 K} \tilde{\mathbf{s}}_{n, r}\left[A\left(\mathbf{e}_{l}\right)\right]_{r}^{T}\left[A\left(\mathbf{e}_{m}\right)\right]_{s} \mathbf{s}_{n, s} \\
& =\sum_{r=1}^{2 K} \sum_{s=1, s \neq r}^{2 K} \mathbf{s}_{n, r}\left(\mathbf{e}_{l}^{T} \mathbf{\Phi}_{r}^{T} \boldsymbol{\Phi}_{s} \mathbf{e}_{m}\right) \mathbf{s}_{n, s} \\
& +\sum_{r=1}^{2 K} \mathbf{s}_{n, r}\left(\mathbf{e}_{l}^{T} \frac{\mathbf{I}_{2 M N}}{\mathbf{\Phi}_{r}^{T} \boldsymbol{\Phi}_{r}} \mathbf{e}_{m}\right) \mathbf{s}_{n, r} \\
& =\sum_{r=1}^{2 K} \sum_{s=1, s \neq r}^{2 K} \mathbf{s}_{n, r}\left(\mathbf{e}_{l}^{T} \mathbf{\Phi}_{r}^{T} \boldsymbol{\Phi}_{s} \mathbf{e}_{m}\right) \mathbf{s}_{n, s}+\sum_{r=1}^{2 K} \mathbf{s}_{n, r} \overbrace{\mathbf{e}_{l}^{T}}^{\mathbf{0}} \mathbf{e}_{m} \mathbf{s}_{n, r} \\
& =-\sum_{r=1}^{2 K} \sum_{s=1, s \neq r}^{2 K} \mathbf{s}_{n, s}\left(\mathbf{e}_{l}^{T} \boldsymbol{\Phi}_{s}^{T} \mathbf{\Phi}_{r} \mathbf{e}_{m}\right) \mathbf{s}_{n, r}+0 \\
& =\sum_{s=1}^{2 K} \sum_{r=1, r \neq s}^{2 K} \mathbf{s}_{n, s}\left(\mathbf{e}_{l}^{T} \boldsymbol{\Phi}_{s}^{T} \boldsymbol{\Phi}_{r} \mathbf{e}_{m}\right) \mathbf{s}_{n, r} \\
& =-\mathbf{s}_{n}^{T} A^{T}\left(\mathbf{e}_{l}\right) A\left(\mathbf{e}_{m}\right) \mathbf{s}_{n}
\end{aligned}
$$

where $\mathbf{s}_{n, r}$ is the $r$ th element of $\mathbf{s}_{n}$. Therefore, we obtain that, for $l \neq m$

$$
\mathbf{s}_{n}^{T} A^{T}\left(\mathbf{e}_{l}\right) A\left(\mathbf{e}_{m}\right) \mathbf{s}_{n}=0
$$

It follows from (24) and (25) that $\mathbf{B}^{T}\left(\mathbf{s}_{n}\right) \mathbf{B}\left(\mathbf{s}_{n}\right)=\|\mathbf{s}(n)\|^{2} \mathbf{I}_{2 M N}$ and the proof is complete. 
It follows from (16) and (18) that given $\mathbf{s}_{n}$, the ML estimate of the channel vector $\mathbf{h}(n)$ can be obtained as

$$
\mathbf{h}_{\mathrm{ML}}(n)=\frac{1}{\|\mathbf{s}(n)\|^{2}} \mathbf{B}^{T}\left(\mathbf{s}_{n}\right) \mathbf{y}_{n}
$$

Therefore, if the information symbols were available, the optimal ML channel estimation would involve a linear estimator as in (26). However, in practice, the information symbols are not available and they have to be estimated. To overcome this problem, one can use an iterative decision-directed channel estimation scheme. That is, given an initial channel estimate for the $n$th block, say $\mathbf{h}^{(0)}(n)$, one can replace $\mathbf{h}(n)$ in (13) with $\mathbf{h}^{(0)}(n)$ and obtain an estimate for $\mathbf{s}_{n}$, say $\hat{\mathbf{s}}_{n}^{(0)}$. This estimate of $\mathbf{s}_{n}$ will, in turn, be used in (26) instead of $\mathbf{s}(n)$ to obtain a new estimate for $\mathbf{h}(n)$, say $\mathbf{h}^{(1)}(n)$. This new channel estimate will again be used in (13) instead of $\mathbf{h}(n)$ to obtain a new estimate of $\mathbf{s}(n)$. This procedure is repeated until the normalized difference between two consecutive channel estimates is negligible. The accuracy of this iterative decision-directed channel estimation scheme depends on the availability of a precise enough initial channel vector estimate $\mathbf{h}^{(0)}(n)$. The proposal here is to use Kalman filtering to obtain the initial channel estimate, $\mathbf{h}^{(0)}(n)$, based on the channel estimates obtained for the previous blocks as well as the $n$th block received data. In what follows, the details of the Kalman filtering technique when applied to our MIMO channel tracking problem are discussed. It is shown that using Lemma 1, the Kalman filter can be simplified significantly. To show this, (16) is used as the observation model of the Kalman filter (Bar-Shalom et al., 2002). Note that the data model in (16) is real-valued. To obtain a real-valued state transition equation, (15) can be rewritten as

$$
\mathbf{h}(n)=\mathbf{F h}(n-1)+\mathbf{w}(n)
$$

where

$$
\mathbf{F} \triangleq\left[\begin{array}{cc}
\operatorname{Re}(\alpha) \mathbf{I}_{M N} & -\operatorname{I} m(\alpha) \mathbf{I}_{M N} \\
\operatorname{I} m(\alpha) \mathbf{I}_{M N} & \operatorname{Re}(\alpha) \mathbf{I}_{M N}
\end{array}\right]
$$

and $\mathbf{w}(n)=\mathbf{W}(n)$ is the real-valued process noise with covariance matrix $\mathbf{Q}=\left(\sigma_{w}^{2} / 2\right) \mathbf{I}_{2 M N}$. We can use $\overline{(27)}$ as the real-valued state transition equation required for Kalman filtering.

The Kalman filtering problem for channel tracking in OSTBC-based MIMO communication system can now be formally stated as follows: Given the measurement-to-state matrix $\mathbf{B}\left(\mathbf{s}_{n}\right)$, use the observed data $\mathbf{y}_{n}$ to find the minimum mean squared error (MMSE) estimate of the components of the state vector $\mathbf{h}(n)$ for each $n \geq 1$.

Given the estimate of the state at time $n-1$, i.e., $\mathbf{h}(n-1 \mid n-1)$, and the associated error covariance matrix $\mathbf{P}(n-1 \mid n-1)$, the Kalman filter (Bar-Shalom et al., 2002) is used to obtain the estimate of the state at time $n$, i.e., $\mathbf{h}(n \mid n)$ and the associated error covariance matrix $\mathbf{P}(n \mid n)$. The Kalman filtering algorithm can be summarized as follows:

$$
\begin{gathered}
\mathbf{h}(n \mid n-1)=\mathbf{F h}(n-1 \mid n-1) \\
\mathbf{P}(n \mid n-1)=\mathbf{F P}(n-1 \mid n-1) \mathbf{F}^{T}+\mathbf{Q}
\end{gathered}
$$




$$
\begin{gathered}
\mathbf{y}_{n}=\mathbf{B}\left(\mathbf{s}_{n}\right) \mathbf{h}(n \mid n-1) \\
\mathbf{v}(n)=\mathbf{y}_{n}-\mathbf{y}_{n} \\
\mathbf{P}_{v}(n)=\mathbf{R}+\mathbf{B}\left(\mathbf{s}_{n}\right) \mathbf{P}(n \mid n-1) \mathbf{B}^{T}\left(\mathbf{s}_{n}\right) \\
\mathbf{G}(n)=\mathbf{P}(n \mid n-1) \mathbf{B}^{T}\left(\mathbf{s}_{n}\right) \mathbf{P}_{v}^{-1}(n) \\
\mathbf{h}(n \mid n)=\mathbf{h}(n \mid n-1)+\mathbf{G}(n) \mathbf{v}(n) \\
\mathbf{P}(n \mid n)=\mathbf{P}(n \mid n-1)-\mathbf{G}(n) \mathbf{P}_{v}(n) \mathbf{G}^{T}(n)
\end{gathered}
$$

where $\mathbf{h}(n \mid n-1)$ is the predicted state, $\mathbf{P}(n \mid n-1)$ is the covariance matrix of the predicted state, $\mathbf{y}_{n}$ is the predicted observation, $\mathbf{v}(n)$ is the innovation process, $\mathbf{P}_{v}(n)$ is the innovation covariance matrix, $\mathbf{G}(n)$ is the Kalman gain (Bar-Shalom et al., 2002), and $\mathbf{R}=E\left\{\mathbf{v}_{n} \mathbf{v}_{n}^{T}\right\}$ is the covariance matrix of the measurement noise $\mathbf{v}_{n}$. As we assumed that the measurement noise is spatio-temporally white with a variance of $\sigma_{v}^{2} / 2$ per real dimension, $\mathbf{R}=\left(\sigma_{v}^{2} / 2\right) \mathbf{I}_{2 M T}$ holds true.

The following Lemma uses the result of Lemma 1 to reduce the computational complexity of finding $\mathbf{P}_{v}^{-1}(n)$ in (34).

Lemma 2: If $\mathbf{P}(n-1 \mid n-1)$ is a diagonal matrix, then, $\mathbf{P}(n \mid n-1)$ in (30) and $\mathbf{P}(n \mid n)$ in (36) are also diagonal, i.e., if

then

$$
\mathbf{P}(n-1 \mid n-1)=\delta_{n-1} \mathbf{I}_{2 M N}
$$

$$
\begin{gathered}
\mathbf{P}(n \mid n-1)=\beta_{n} \mathbf{I}_{2 M N} \\
\mathbf{P}(n \mid n)=\delta_{n} \mathbf{I}_{2 M N}
\end{gathered}
$$

where

$$
\beta_{n}=\delta_{n-1}|\alpha|^{2}+\frac{\sigma_{w}^{2}}{2} \quad \text { and } \quad \delta_{n}=\frac{\sigma_{v}^{2} \beta_{n}}{2\|s(n)\|^{2} \beta_{n}+\sigma_{v}^{2}} .
$$

Proof: Substituting (37) into the predicted state in (30), we can rewrite it as

$$
\mathbf{P}(n \mid n-1)=\delta_{n-1} \mathbf{F F}^{T}+\mathbf{Q}=|\alpha|^{2} \delta_{n-1} \mathbf{I}_{2 M N}+\mathbf{Q}=\underbrace{\left(|\alpha| \delta_{n-1}^{2}+\frac{\sigma_{w}^{2}}{2}\right)}_{\beta_{n}} \mathbf{I}_{2 M N} .
$$

Inserting (41) into (33) and using matrix inversion lemma, $\mathbf{P}_{v}^{-1}(n)$ in (33) can be written as

$$
\begin{aligned}
\mathbf{P}_{v}^{-1}(n) & =\mathbf{R}^{-1}-\mathbf{R}^{-1} \mathbf{B}\left(\mathbf{s}_{n}\right)\left(\mathbf{B}^{T}\left(\mathbf{s}_{n}\right) \mathbf{R}^{-1} \mathbf{B}\left(\mathbf{s}_{n}\right)+\mathbf{P}^{-1}(n \mid n-1)\right)^{-1} \mathbf{B}^{T}\left(\mathbf{s}_{n}\right) \mathbf{R}^{-1} \\
& =\frac{2}{\sigma_{v}^{2}} \mathbf{I}_{2 M T}-\frac{4}{\sigma_{v}^{4}} \mathbf{B}\left(\mathbf{s}_{n}\right)\left(\frac{2}{\sigma_{v}^{2}} \mathbf{B}^{T}\left(\mathbf{s}_{n}\right) \mathbf{B}\left(\mathbf{s}_{n}\right)+\frac{1}{\beta_{n}} \mathbf{I}_{2 M N}\right)^{-1} \mathbf{B}^{T}\left(\mathbf{s}_{n}\right)
\end{aligned}
$$




$$
=\frac{2}{\sigma_{v}^{2}} \mathbf{I}_{2 M T}-\left(\frac{4 \beta_{n}}{2\|\mathbf{s}(n)\|^{2} \beta_{n} \sigma_{v}^{2}+\sigma_{v}^{4}}\right) \mathbf{B}\left(\mathbf{s}_{n}\right) \mathbf{B}^{T}\left(\mathbf{s}_{n}\right)
$$

where the fact that $\mathbf{B}^{T}\left(\mathbf{s}_{n}\right) \mathbf{B}\left(\mathbf{s}_{n}\right)=\|\mathbf{s}(n)\|^{2} \mathbf{I}_{2 M N}$ has been used.

Using (34) and (42), we rewrite (36) as

$$
\begin{gathered}
\mathbf{P}(n \mid n)=\mathbf{P}(n \mid n-1)-\mathbf{P}(n \mid n-1) \mathbf{B}^{T}\left(\mathbf{s}_{n}\right) \\
\left(\frac{2}{\sigma_{v}^{2}} \mathbf{I}_{2 M T}-\left(\frac{4 \beta_{n}}{2\|\mathbf{s}(n)\|^{2} \beta_{n} \sigma_{v}^{2}+\sigma_{v}^{4}}\right) \mathbf{B}\left(\mathbf{s}_{n}\right) \mathbf{B}^{T}\left(\mathbf{s}_{n}\right)\right) \mathbf{B}\left(\mathbf{s}_{n}\right) \mathbf{P}(n \mid n-1) \\
=\left(\frac{\sigma_{v}^{2} \beta_{n}}{2\|\mathbf{s}(n)\|^{2} \beta_{n}+\sigma_{v}^{2}}\right) \mathbf{I}_{2 M N} .
\end{gathered}
$$

The proof is complete.

Based on Lemma 2, if $\mathbf{P}(0 \mid 0)$ is initialized as a diagonal matrix, $\mathbf{P}(n \mid n-1)$ and $\mathbf{P}(n \mid n)$ always take the form of (38) and (39), respectively. Hence, $\mathbf{P}_{v}^{-1}$ in (34) is simplified as in (42). It is also noteworthy that using (41) and (42) the Kalman filter gain $\mathbf{G}(n)$ in (34) can be written as

$$
\mathbf{G}(n)=\underbrace{\beta_{n}\left(\frac{2}{\sigma_{v}^{2}}-\frac{4 \beta_{n}\|\mathbf{s}(n)\|^{2}}{2\|\mathbf{s}(n)\|^{2} \beta_{n} \sigma_{v}^{2}+\sigma_{v}^{4}}\right)}_{\stackrel{\Delta}{=} \mu_{n}} B^{T}\left(\mathbf{s}_{n}\right) .
$$

Using (31), (32) and (44), we can simplify (35) as

$$
\begin{aligned}
\mathbf{h}(n \mid n) & =\mathbf{h}(n \mid n-1)+\mu_{n} \mathbf{B}^{T}\left(\mathbf{s}_{n}\right)\left(\mathbf{y}(n)-\mathbf{B}\left(\mathbf{s}_{n}\right) \mathbf{h}(n \mid n-1)\right) \\
& =\left(1-\mu_{n}\|\mathbf{s}(n)\|^{2}\right) \mathbf{h}(n \mid n-1)+\mu_{n} \mathbf{B}^{T}\left(\mathbf{s}_{n}\right) \mathbf{y}(n) .
\end{aligned}
$$

Therefore, the Kalman filtering algorithm presented in (29)--(36) can be simplified as it follows:

$$
\begin{gathered}
\mathbf{h}(n \mid n-1)=\mathbf{F h}(n-1 \mid n-1) \\
\beta_{n}=\delta_{n-1}|\alpha|^{2}+\frac{\sigma_{w}^{2}}{2}, \quad \mu_{n}=\beta_{n}\left(\frac{2}{\sigma_{v}^{2}}-\frac{4 \beta_{n}\|\mathbf{s}(n)\|^{2}}{2\|\mathbf{s}(n)\|^{2} \beta_{n} \sigma_{v}^{2}+\sigma_{v}^{4}}\right) \\
\mathbf{h}^{(0)}(n)=\mathbf{h}(n \mid n)=\left(1-\mu_{n}\|\mathbf{s}(n)\|^{2}\right) \mathbf{h}(n \mid n-1)+\mu_{n} \mathbf{B}^{T}\left(\mathbf{s}_{n}\right) \mathbf{y}(n) \\
\delta_{n}=\frac{\sigma_{v}^{2} \beta_{n}}{2\|\mathbf{s}(n)\|^{2} \beta_{n}+\sigma_{v}^{2}} .
\end{gathered}
$$

We then use the so-obtained $\mathbf{h}^{(0)}(n)$ in the aforementioned iterative procedure to improve its accuracy. 
Remark 1: Note that the simplified Kalman filter requires the knowledge of the symbol vector $\mathbf{s}_{n}$ (or $\left.\mathbf{B}(\mathbf{s}(n))\right)$. However, the primary objective is to decode $\mathbf{s}(n)$. To overcome this obstacle, we propose to replace $\mathbf{s}_{n}$ in the Kalman filter equations (46)--(49), by its estimate, which is obtained by replacing the true channel vector in (13) by the predicted channel vector $\mathbf{h}(n \mid n-1)$ as

$$
\mathbf{s}_{n}=\frac{1}{\|\mathbf{h}(n \mid n-1)\|^{2}} A^{T}(\mathbf{h}(n \mid n-1)) \mathbf{y}_{n} .
$$

Note that given the predicted channel vector $\mathbf{h}(n \mid n-1)$, the symbol estimate in (50) is optimal in the ML sense.

Remark 2: To initiate the whole process, we also need to obtain an accurate channel estimate $\mathbf{h}(0)$ as well as its initial covariance $\delta_{0} \mathbf{I}_{2 M N}$. To obtain such an initial channel estimate, one can use a training block $\mathbf{s}(0)$, which is known at the receiver. At the beginning of the tracking process, the receiver can then use (26) to obtain the ML estimate of $\mathbf{h}(0)$ as

$$
\mathbf{h}(0)=\frac{1}{\|\mathbf{s}(0)\|^{2}} \mathbf{B}^{T}\left(\mathbf{s}_{0}\right) \mathbf{y}_{0}
$$

where $\mathbf{s}_{0}=\underline{\mathbf{s}(0)}$ is defined.

To find $\delta_{0}$, we note that

$$
\begin{gathered}
\delta_{0} \mathbf{I}_{2 M N}=E\left\{(\mathbf{h}(0)-\hat{\mathbf{h}}(0))(\mathbf{h}(0)-\hat{\mathbf{h}}(0))^{T}\right\} \\
=E\left\{\mathbf{h}(0) \mathbf{h}^{T}(0)\right\}+E\left\{\mathbf{h}(0) \mathbf{h}^{T}(0)\right\}-2 E\left\{\mathbf{h}(0) \mathbf{h}^{T}(0)\right\}
\end{gathered}
$$

where channel is assumed zero-mean and

$$
\begin{aligned}
E\left\{\mathbf{h}(0) \mathbf{h}^{T}(0)\right\} & =\frac{1}{\|\mathbf{s}(0)\|^{4}} \mathbf{B}^{T}\left(\mathbf{s}_{0}\right) E\left\{\mathbf{y}_{0} \mathbf{y}_{0}^{T}\right\} \mathbf{B}\left(\mathbf{s}_{0}\right) \\
& =\frac{1}{\|s(0)\|^{4}} \mathbf{B}^{T}\left(\mathbf{s}_{0}\right)(\mathbf{B}\left(\mathbf{s}_{0}\right) \underbrace{E\left\{\mathbf{h}(0) \mathbf{h}^{T}(0)\right\}}_{\left(\sigma_{h}^{2} / 2\right) \mathbf{I}_{2 M N}} \mathbf{B}^{T}\left(\mathbf{s}_{0}\right)+\left(\sigma_{v}^{2} / 2\right) \mathbf{I}_{2 M T}) \mathbf{B}\left(\mathbf{s}_{0}\right) \\
& =\left(\frac{\sigma_{h}^{2}}{2}+\frac{\sigma_{v}^{2}}{2\|\mathbf{s}(0)\|^{2}}\right) \mathbf{I}_{2 M N} \\
& =\frac{1}{\|\mathbf{s}(0)\|^{2}} \mathbf{B}^{T}\left(\mathbf{s}_{0}\right) E\left\{\left(\mathbf{B}\left(\mathbf{s}_{0}\right) \mathbf{h}(0)+\mathbf{v}_{0}\right) \mathbf{h}^{T}(0)\right\}=\frac{\sigma_{h}^{2}}{2} \mathbf{I}_{2 M N}
\end{aligned}
$$

Therefore, one can obtain $\delta_{0}$ as

$$
\delta_{0}=\frac{1}{2} \frac{\sigma_{v}^{2}}{\|\mathbf{s}(0)\|^{2}} .
$$


Remark 3: To avoid error propagation, training should be repeated at regular intervals. The training repetition period (TRP) determines the bandwidth efficiency of the system and it is defined as the distance, in terms of number of blocks, between two consecutive training blocks.

Remark 4: In terms of computational complexity, the proposed channel tracking method enjoys the low computational complexity of linear processing. More specifically, the first step requires the computation of $\mathbf{B}^{T}\left(\mathbf{s}_{n}\right) \mathbf{y}(n)$, and therefore, $2 M T$ real multiplications are required for computation of each entry of $\mathbf{h}(n \mid n)$. Taking into account that $\mathbf{h}(n \mid n)$ is of length $2 M N$, the total computational complexity of the first step is of order $O\left(M^{2} N T\right)$. The second step is indeed an iterative algorithm. In each iteration, we need to compute four quantities: $\left\|\mathbf{h}^{(k-1)}(n)\right\|^{2}, \quad \mathbf{A}^{T}\left(\mathbf{h}^{(k-1)}(n)\right) \mathbf{y}_{n},\left\|\mathbf{s}_{n}^{k}\right\|^{2}$, and $\mathbf{B}^{T}\left(\mathbf{s}_{n}^{(k)}\right) \mathbf{y}_{n}$. Computing these four quantities requires $2 M N, 4 K M T, 2 K$, and $4 M^{2} N T$ real multiplications, respectively. Therefore, the computational complexity of the second step is of the order $O\left(M^{2} N T\right)$ per iteration of the first step. The traditional Kalman filtering method involves the computation of $\mathbf{P}_{v}^{-1}(n)$. This amounts to a computational complexity of the order $O\left(M^{3} T^{3}\right)$ per iteration. Therefore, the proposed method significantly reduces the computational complexity of the traditional Kalman filtering.

\section{Simulation results}

In our numerical example, we consider the $3 / 4$ rate code of (Larsson \& Stoica, 2003) with $N=M=T=4$, and $K=3$. The SNR is defined as $\sigma_{h}^{2} / \sigma_{v}^{2}$. In each simulation run, the elements of $H(n)$ are generated according to Jakes model (Jakes Jr, 1974) corresponding to $F_{m} T_{s}=0.0045$ where $F_{m}$ is the doppler frequency and $T_{s}$ is the sampling time. This results in $\alpha=J_{0}\left(0.2 \pi F_{m} T_{s}\right) e^{j 2 \pi F_{o} T_{s}}=0.9998 e^{j 0.0283}$ where $J_{0}(\cdot)$ is the zeroth order Bessel function of first kind. In terms of channel estimation accuracy, we compare our Kalman filtering based channel tracking technique with the online implementation of the technique developed in (Shahbazpanahi et al., 2005). In order to implement the method of (Shahbazpanahi et al., 2005) in an online manner, we have used the subspace tracking approach proposed in section III.G of (Shahbazpanahi et al., 2005). In our comparison, we use normalized mean squared error (NMSE) of the channel estimates defined as

$$
\mathbf{N M S E}=E\left\{\frac{\|\mathbf{H}(n)-\widehat{\mathbf{H}}(n)\|^{2}}{\|\mathbf{H}(n)\|^{2}}\right\} .
$$

In terms of symbol error rate (SER), we compare our method not only with the method of (Shahbazpanahi et al., 2005) but also with the differential space-time coding scheme (Larsson \& Stoica, 2003).

Figure 1 illustrates the SERs of different methods, versus SNR, for TRP $=10$. In this figure, we have also plotted the SER for the (clairvoyant) coherent ML receiver that is aware of the time-varying channel. It is noteworthy that the latter receiver does not correspond to any practical application and it is herein considered only for the sake of comparison. We have 
also plotted the performance of a differential coding scheme which uses the same OTSTBC which we have used in our method. As can be seen from this figure, for TRP $=10$, our Kalman filtering based technique outperforms the differential space-time coding scheme by $1 \mathrm{~dB}$.

It is worthwhile to observe that our technique outperforms the technique of (Shahbazpanahi et al., 2005) by more than $2 \mathrm{~dB}$. In fact, when applied to track a time-varying MIMO channel, the algorithm of (Shahbazpanahi et al., 2005) performs even worse than the differential scheme. This is not surprising as (Shahbazpanahi et al., 2005) assumes that the MIMO channel is fixed within the observation interval. Therefore, the method of (Shahbazpanahi et al., 2005) is not applicable whenever the MIMO channel variations are fairly fast.

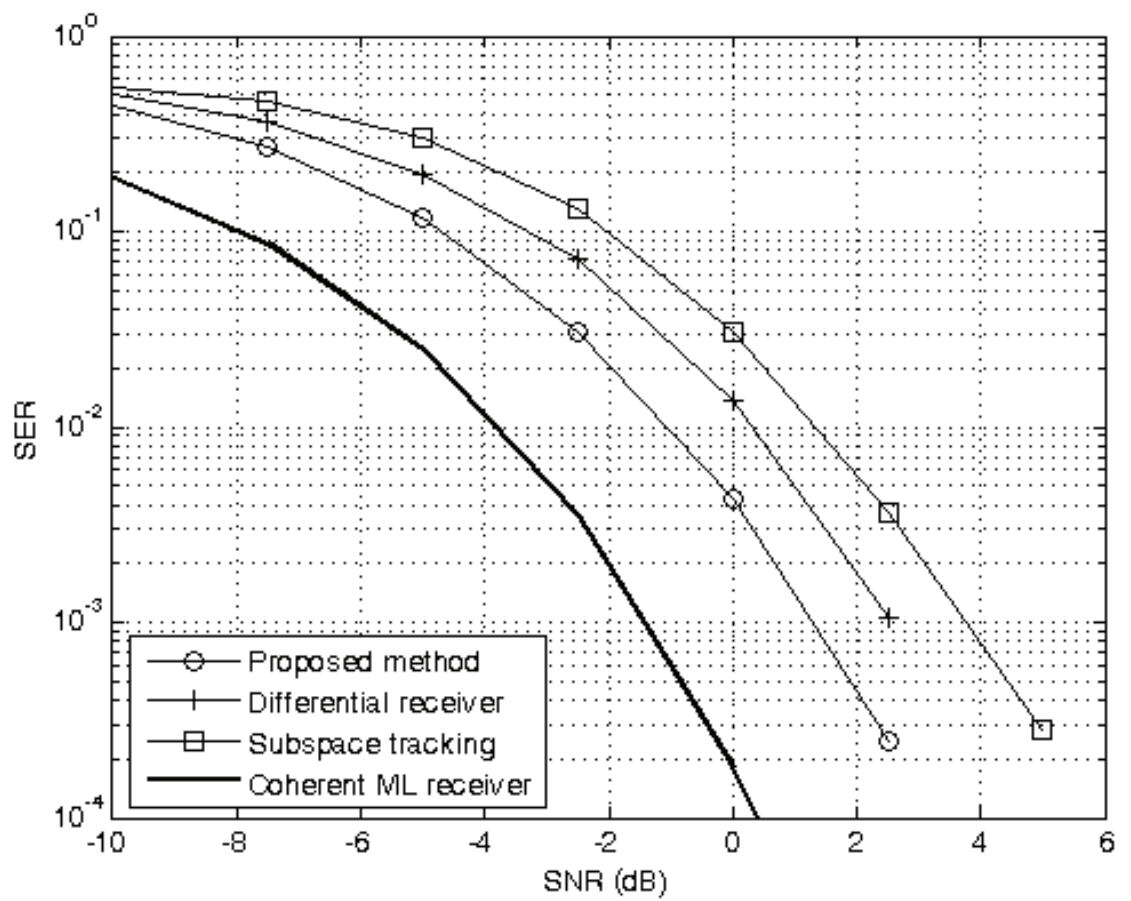

Fig. 1. The SERs versus SNR for different methods and for TRP $=10$.

\section{Conclusions}

In this Chapter, an efficient Kalman filtering solution for the problem of channel tracking in MIMO communication systems where the MIMO channel is time-varying is presented. Considering the type of MIMO systems where orthogonal space-time block codes are used to encode the information symbols a two-step MIMO channel tracking algorithm is presented. As the first step, Kalman filtering is used to obtain an initial channel estimate for the current block based on the channel estimates obtained for previous blocks. In the second step, the so-obtained initial channel estimate is refined using a decision-directed iterative 
method. In is shown that due to the interesting properties of orthogonal space-time block codes, both the Kalman filter and the decision-directed algorithm can be significantly simplified.

\section{References}

Al-Naffouri, T., Awoniyi, O., Oteri, O., and Paulraj, A. (2004). Receiver design for MIMOOFDM transmission over time variant channels, IEEE Global Telecommunications Conference, volume 4, pages 2487-2492.

Alamouti, S. (1998). A simple transmit diversity technique for wireless communications. IEEE Journal on Selected Areas in Communications, Vol. 16, No. 8, pp. 1451-1458.

Balakumar, B., Shahbazpanahi, S., and Kirubarajan, T. (2007), Joint MIMO channel tracking and symbol decoding using Kalman filtering, IEEE Transactions on Signal Processing, Vol. 55, No. 12, pp. 5873-5879.

Bar-Shalom, Y., Kirubarajan, T., and Li, X.-R. (2002), Estimation with Applications to Tracking and Navigation, John Wiley \& Sons, Inc., New York.

Coon, J., Armour, S., Beach, M., and McGeehan, J. (2005), Adaptive frequency-domain equalization for single-carrier multiple-input multiple-output wireless transmissions, IEEE Transactions on Signal Processing, Vol. 53, No. 8, pp. 32473256.

Ganesan, G. and Stoica, P. (2001), Space-time block codes: a maximum SNR approach, IEEE Transactions on Information Theory, Vol. 47, No. 4, pp. 1650-1656.

Gharavi-Alkhansari, M. and Gershman, A. B. (2005), Constellation space invariance of space-time block codes, IEEE Transactions on Information Theory, Vol. 51, No. 1, pp. 331-334.

Haykin, S., Huber, K., and Chen, Z. (2004), Bayesian sequential state estimation for MIMOwireless communications, Proceedings of the IEEE, Vol. 92, No. 3, pp. 439-454.

Jakes Jr, W. (1974). Microwave Moble Communication. John Wiley \& Sons, Inc., New York.

Larsson, E. G. and Stoica, P. (2003), Space-time block coding for wireless communications, Cambridge UniversityPress.

Liu, Z., Ma, X., and Giannakis, G. (2002), Space-time coding and Kalman filtering for timeselective fading channels, IEEE Transactions on Communications, Vol. 50, No. 2, pp. 183-186.

Schafhuber,D.,Matz, G., and Hlawatsch, F. (2003), Kalman tracking of time-varying channels in wireless MIMO-OFDM systems, In Thirty-Seventh Asilomar Conference on Signals, Systems and Computers, Vol. 2, pp. 1261-1265.

Shahbazpanahi, S., Gershman, A., and Manton, J. (2005), Closed-form blind MIMO channel estimation for orthogonal space-time block codes, IEEE Transactions on Signal Processing, Vol. 53, No. 12, pp. 4506-4517.

Tarokh, V., Jafarkhani, H., and Calderbank, A. (1999), Space-time block codes from orthogonal designs, IEEE Transactions on Information Theory, Vol. 45, No. 5, pp. 1456-1467.

Tsatsanis, M., Giannakis, G., and Zhou, G. (1996), Estimation and equalization of fading channels with random coefficients, IEEE International Conference on Acoustics, Speech, and Signal Processing, Vol. 2, pp. 1093-1096. 
Wang, H. S. and Chang, P.-C. (1996), On verifying the first-order Markovian assumption for a Rayleigh fading channel model, IEEE Transactions on Vehicular Technology, Vol. 45, No. 2, pp. 353-357. 


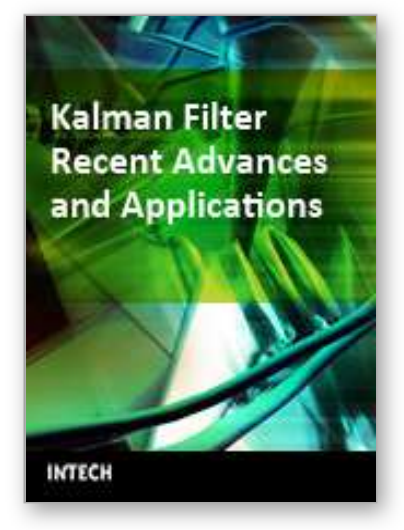

\section{Kalman Filter Recent Advances and Applications \\ Edited by Victor M. Moreno and Alberto Pigazo}

ISBN 978-953-307-000-1

Hard cover, 584 pages

Publisher InTech

Published online 01, April, 2009

Published in print edition April, 2009

The aim of this book is to provide an overview of recent developments in Kalman filter theory and their applications in engineering and scientific fields. The book is divided into 24 chapters and organized in five blocks corresponding to recent advances in Kalman filtering theory, applications in medical and biological sciences, tracking and positioning systems, electrical engineering and, finally, industrial processes and communication networks.

\section{How to reference}

In order to correctly reference this scholarly work, feel free to copy and paste the following:

B. Balakumar and T. Kirubarajan (2009). Joint MIMO Channel Tracking and Symbol Decoding, Kalman Filter Recent Advances and Applications, Victor M. Moreno and Alberto Pigazo (Ed.), ISBN: 978-953-307-000-1, InTech, Available from:

http://www.intechopen.com/books/kalman_filter_recent_adavnces_and_applications/joint_mimo_channel_track ing_and_symbol_decoding

\section{INTECH}

open science | open minds

\section{InTech Europe}

University Campus STeP Ri

Slavka Krautzeka 83/A

51000 Rijeka, Croatia

Phone: +385 (51) 770447

Fax: +385 (51) 686166

www.intechopen.com

\section{InTech China}

Unit 405, Office Block, Hotel Equatorial Shanghai

No.65, Yan An Road (West), Shanghai, 200040, China

中国上海市延安西路65号上海国际贵都大饭店办公楼 405 单元

Phone: +86-21-62489820

Fax: $+86-21-62489821$ 
(C) 2009 The Author(s). Licensee IntechOpen. This chapter is distributed under the terms of the Creative Commons Attribution-NonCommercialShareAlike-3.0 License, which permits use, distribution and reproduction for non-commercial purposes, provided the original is properly cited and derivative works building on this content are distributed under the same license. 